\title{
A Digital Watermarking Algorithm Based on Wavelet Packet Transform and RBF Neural Network \\ Poorvi Saxena ${ }^{1}$, Murlidhar Vishwakarma ${ }^{2}$ \\ Poorvisaxena10@gmail.com¹, murli.mtech@gmail.com² \\ Department of Computer Science \& Engineering ${ }^{1,2}$ \\ REC, Bhopal, India
}

\begin{abstract}
Digital water marking technique suffered some problem of geometrical and some other attack. The process of attack deformed the quality of digital image and violet the rule of copyright protection low. For the roughness of digital image watermarking used wavelet transform function and RBF neural network. The RBF neural network trained the pattern of digital image pixel and finally embedded the image. The processes of validation of blindness of digital image apply some geometrical attack. Our empirical result computed in form of PSNR value and number of correlation of embedded image. Our evolution process shows better result in compression of transform based digital water marking technique.
\end{abstract}

Key words: - digital watermarking, WPT, RBF network

\section{Council for Innovative Research}

Peer Review Research Publishing System

Journal: INTERNATIONAL JOURNAL OF COMPUTERS \& TECHNOLOGY

Vol 10, No 7 


\section{INTRODUCTION}

Digital image watermarking is a kind of technology that embeds copyright information into multimedia content. An effective image watermarking mainly includes watermark making, watermark embed, watermark discovery, and watermark attack [2], [1]. Digital image watermarking provides copyright protection to image by hiding appropriate information in original image to declare rightful ownership [3]. There are four essential factors those are commonly used to determine quality of watermarking scheme. They are robustness, dilation, strength, and visibility. Robustness is a measure of immunity of watermark against attempts to image modification and manipulation like compression, filtering, rotation, scaling, noise attacks, resizing, cropping etc. Imperceptibility is the quality that the cover image should not be destroyed by the presence of watermark. The basic idea that wavelet transform apply to image processing is decomposed the image by multiresolution, then generate sub-image with different spaces and independent bands, at last, process the coefficients of subimage. The neural network has the characteristics of approaching non-linear mapping, and has good generalization ability. If a neural network was trained in the watermark embedding process, through studying, the Corresponding relations of pixel values between before and after embedding the watermark signal is obtained, then the watermark can be extracted by using the trained neural network, thus the blind detection of the watermarking Algorithm is achieved. In this paper proposed a new algorithm of digital watermarking based on wavelet transform and RBF neural network. The watermark was embedded in the wavelet image which was the host image after discrete wavelet packet transform, so that the watermarking could be embedded into a narrower and finer band, and the transparency and robustness of watermarking could be improved better. This algorithm decomposed the host image by discrete wavelet packet transform. Then watermarking was embedded into the position which was chosen by the key in wavelet image. In the watermarking embedding process, sixteen neighborhood pixels around the embedded point was select as input vector of the RBF neural network. The above section discuss introduction of digital water marking and process of work In section II we describe related work of watermarking. In section III proposed methodology. In section IV discuss experimental result analysis and finally conclude in section V.

\section{II.RELATED WORK}

In this section describe method for digital watermarking based on different approach such wavelet transform function and some optimization technique.

[3] Proposed a novel method for watermarking based on back-propagation neural network and wavelet packet transform. The algorithm first decomposed the host image via discrete wavelet packet transform, then embedded water-marking into the position which was chosen by the key in wavelet image. In the process of embedding watermarking, eight neighborhood pixels around the embedded point was selected as sample input of the BP neural network, and the pixel value of the embedded point was chosen as the ideal output, finally, trained the neural network. Using the trained neural network, blind watermarking extraction was achieved.

[12] Proposed a new method for digital water marking based on frequency quantization for the protection of geometrical attack. In concern of water marking by authors describe the process of embedded technique as Firstly, ununiformity of each unit quaternion block in host image is calculated to pick up the potential block adaptively. Secondly, frequency matrix of host color image is obtained by using quaternion Fourier transforms. After that, the water marks are embedded into the amplitude of AC coefficient. Quaternion looks at color pixels as a holistic vector has the ability to retain the interaction between color spectral channels during the entire image processing and achieves a better tradeoff between robustness and perceptivity.

[11] Proposed a method for Color Image Copyright Protection Digital Watermarking Algorithm Based on DWT \& DCT in this technique authors describe a removal of geometrical attack for watermarking as visual characteristics of low frequency sub-image of DWT and the ability of DCT to remove correlation between DWT coefficients. By doing this, this technique improves a blind DCT watermarking algorithm to get a new color image digital watermarking schema based on DWT and DCT. The schema applies a self-adapted color component choosing strategy; Preprocesses the watermark by Logistic chaotic encryption; Applies the DCT to low frequency sub-image of DWT to get rid of coefficient correlation; In addition to that this schema extract strategy is blind by using sub-sampling technique.

[12] Proposed a method for digital watermarking Algorithm in Discrete Cosine transformation Domain As the replication and modification of digital media content is done frequently and without any significant obstruction, secrecy and authenticity become vulnerable to attacks. In the information hiding community digital watermarking has achieved immense popularity due to its righteous stronghold against piracy and non-repudiation. Many watermarking algorithm has been developed in recent years. From the context of the purposes, as they serve, they differ from each other. Here we propose some basic algorithms of digital watermarking technique using LSB (Least Significant Bit) and DCT (Discrete Cosine Transformation).

[25] Proposed a robust wavelet-based watermarking scheme for copyright protection of digital images as the steps involved in the embedding of watermark image into the original image are presented in this sub-section. In the proposed scheme, a binary image is used as the watermark data. Initially, the original image is subjected to wavelet decomposition using Haar wavelet transform to obtain the four sub-bands $\mathrm{LL}, \mathrm{LH}, \mathrm{HL}$ and $\mathrm{HH}$. For the embedding process, the $\mathrm{HH}$ subband is chosen. Simultaneously, the original image is provided as input to the MD5 algorithm, which results a hash value. The hash value thus obtained is used as seed for a random function which generates a random matrix with the size of the original image. Subsequently, a mask matrix is generated from the generated random matrix with the aid of two relationship degree factors. The binary watermark is embedded into the $\mathrm{HH}$ subband by using the generated mask matrix and the watermark strength $\beta$. The resultant will be the $\mathrm{HH}$ sub-band with the watermark embedded in. The modified $\mathrm{HH}$ 
sub-band is mapped back to its original position and then inverse Haar Wavelet Transform is applied, to obtain the watermarked image.

In [27] a robust watermarking approach based upon bidimensional empirical mode decomposition (BEMD) against geometric distortion is proposed. This method uses the orthogonal properties of bi-dimensional empirical mode decomposition (BEMD) to achieve the piece based orthogonal change in the image. This method allows study of nonlinear and non -stationary data. It decomposes a given signal into many frequency components, called intrinsic mode function (IMF). All these decomposed parts of data or image having different frequency. Watermark is embedded into the intermediary frequency IMF. Middle frequency is adoptively weighted on the basis of image visual system and orthogonal transform. Experimentally it clears that this method can show the watermark when the image is half cut and having excellent robustness against image shearing.

In [28] a new watermarking algorithm to confront the geometric transformation based on the feature points is proposed. Feature point is selected using the Robert operator and watermark is embedded in these regions. The algorithm is tested against normal image processing; Photoshop is used to extract the watermarking after JPEG compression with different quality factor. As the value of quality factor value goes down watermark image gets more distorted. For these quality factor Q, NC (Normalized Correlation coefficient) value is inversely proportional it's clear by experiment.

\section{PROPOSED METHODOLOGY}

The watermarking models have proposed provide greater security against sophisticated geometrical attacks in different domains while providing sufficient watermark-carrying capacity at the same time. The distortion is extremely small in the models, thereby making accidental detection of watermark in a random object almost negligible. RBF has been facilitated in the watermarking algorithms and is a solution to the secondary watermarking attacks. RBF is a neural network technique for the improvement of watermark capacity. RBF method has found that trained pattern as the image. While the line between each continues to pixel, these two differences usually still apply. (1) Train pattern (2) embedded train pattern in watermark. There's also a range of more minor differences, the above two are often what most impact one's technique. In the proposed technique we used $\mathrm{YCbCr}$ color model for distribution of color model. The given image in general in RGB format. Change this format into YCbCr. $\mathrm{Y}$ component is luminance component and $\mathrm{Cb}$ corma and $\mathrm{Cr}$ corma. After conversion of color component we apply RBF technique for better compactness of color pixel position. we apply DWT transform technique for watermarking these technique decompose image into horizontal, vertical and diagonal formation of pixel ,so in this process pixel interchange and pattern is important aspect of digital image. That aspect is fulfilling by the RBF technique. The watermark embedding in the DWT by using four-level DWT to an input image $f(x, y)\left(512^{\star} 512^{\star} 16\right.$ bits $)$ generate 12 sub bands of high frequency $(\mathrm{LHi}, \mathrm{HLi}, \mathrm{i}=1-4)$ and one low frequency subband(LL4).the RBF interchange the 16 bit pixel position for watermarking. And interchange the pixel correlation and improve the PSNR value of watermarked image. In this paper proposed a novel methodology for image Watermark based on wavelet transform and radial biases neural network. Initially the discrete wavelet transform function is applied into input image. Now input image decomposed in to layer structure form. After that we calculate horizontal, vertical and diagonal coefficient of input image, after that we apply transform vector technique and generate trained pattern using ACP algorithm. In RBF network we used Gaussian based kernel function. The ACP algorithm generates a trained pattern for the embedded of watermark image. In that process the correlation factor of watermark is increase and the target PSNR value is achieved. As known, the high-order statistical relationship does play an important part in image watermarking technique area.So in order to take advantage of the high-order statistical relationship among variables, so we used ACP algorithm for training the network. Proposed Watermark is a three-layer neural network with inputs derived from an NxN neighborhood of the transformed image and appropriately selected neuron activation functions. As shown in Figure 1, the network takes $Y p$ and $\Delta Y k$ as the inputs, where $\mathrm{Yp}$ is the wavelet transform coefficient under consideration, which is the center of a $\mathrm{N} \times \mathrm{N}$ processing window, and $\Delta Y k=Y k-Y p$ is the difference value between $Y p$ and the coefficient $Y k(k=0,1, \ldots, N 2-1, k \Delta p)$ of the other points in the $N \times N$ window. Figure 2 shows an example of a processing window with a size of $3 \times 3$ pixels. In this example, Y12 is the center of the window, and $\Delta \mathrm{Yk} Y 12(\mathrm{k}=0,1, \ldots, 24, \mathrm{k} \Delta 12)$.ablest, so we used ACP algorithm for training the network.

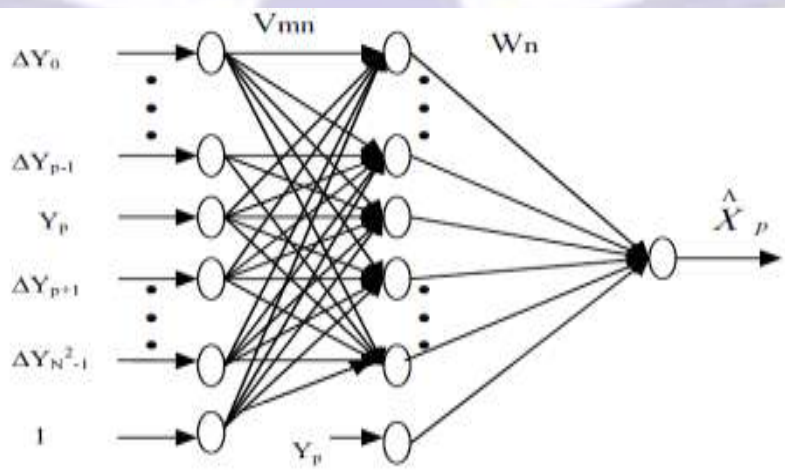

Figure 1Neural network structure 


\begin{tabular}{|l|l|l|}
\hline Y0 & Y1 & Y2 \\
\hline Y3 & Y4 & Y5 \\
\hline Y6 & Y7 & Y9 \\
\hline & & \\
\hline
\end{tabular}

Figure 2 shows that unit input vector pixel the output of network is linear activation function.that activation function perform the targeted output of PSNR value.

Step for proposed methodology.

1. Input host and water mark image.

2. Perform wavelet transform and image decomposed in layers.

3. Find horizontal, vertical and diagonal coefficient of wavelet.

4. Apply soft thresholding of wavelet

5. Check value of coefficient of wavelet

6. Decide the size of vector input $3^{\star} 3$

7. Trained the network.

8. Apply target value of activation function

9. Find PSNR with variance

10. Image Watermark result.

\section{EXPERIMENTAL RESULT ANALYSIS}

The proposed watermarking algorithms are implemented using MATLAB. The imperceptibility and the robustness of the watermarked image are tested with PSNR and NC. Three family images and one Lena image of size512x512 is selected as the cover image. Color image of size $64 \times 64$ local is selected as the watermark. The PSNR of the watermarked image is calculated using the formula

$$
P S N R=10 \log _{10}^{\frac{(R * R)}{M S E}}
$$

Where $\mathrm{R}$ is maximum fluctuation in the cover image $=511$

$M S E=\sum_{j=1}^{r} \sum_{k=1}^{c} \frac{W(j, k)-W^{\prime}(j, k)}{r c}$

Where

$r=$ number of rows in the digital image $c=$ number of columns in digital image $w(j, k)=$ cover image $w(j, k)=$ cover image

$N C=\frac{\sum_{j} \sum_{k} W(j, k) * W^{\prime}(j, k)}{\sum_{j} \sum_{k} W(j, k) * W(j, k)}$

The performance evaluation of the methods is done by measuring imperceptibility and robustness. The normalized correlation coefficient (NC) is used to measure the similarity between the cover image and the watermarked image. Peak Signal-to-Noise Ratio (PSNR) is used to measure the imperceptibility of the watermarked image. The robustness of the watermarked image is tested by attacks such as JPEG compression, cropping, median filtering, salt \& pepper noise attack, and rotation. 

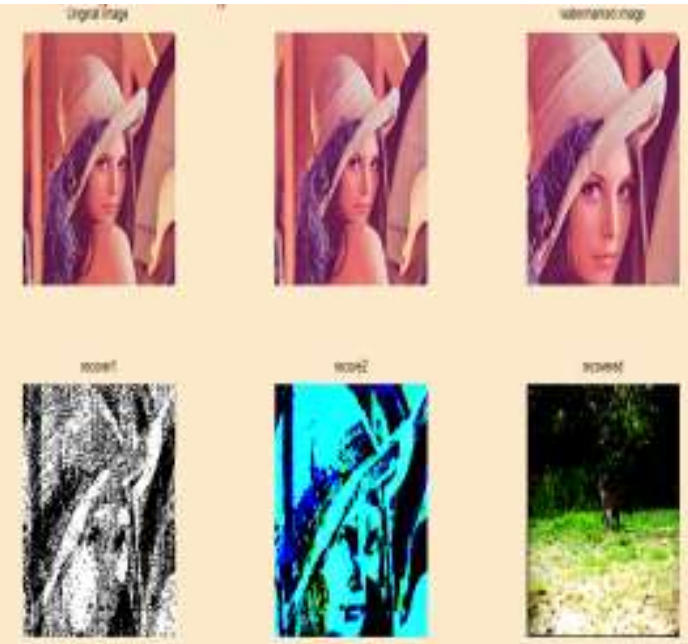

Figure 3 indicated that there were no visual differences between host image and the image embedded Watermark. And the watermarking had good imperceptibility. The peak signal to noise ratio was PSNR $=22.2035$, the extracted watermark and the original watermarking similarity was $\mathrm{NC}=0.4369$
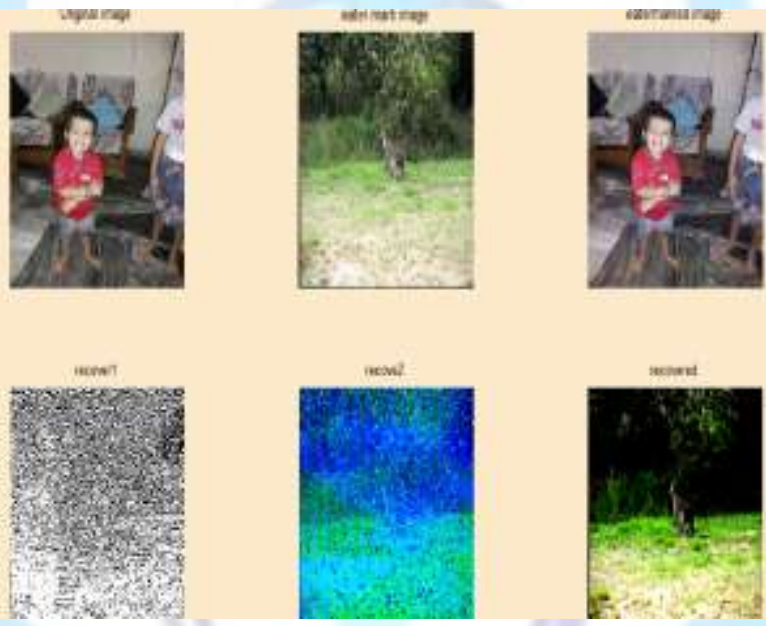

Figure 4 indicated that there were no visual differences between host image and the image embedded Watermark. And the watermarking had good imperceptibility. The peak signal to noise ratio was PSNR $=24.2035$, the extracted watermark and the original watermarking similarity was $\mathrm{NC}=.5678$
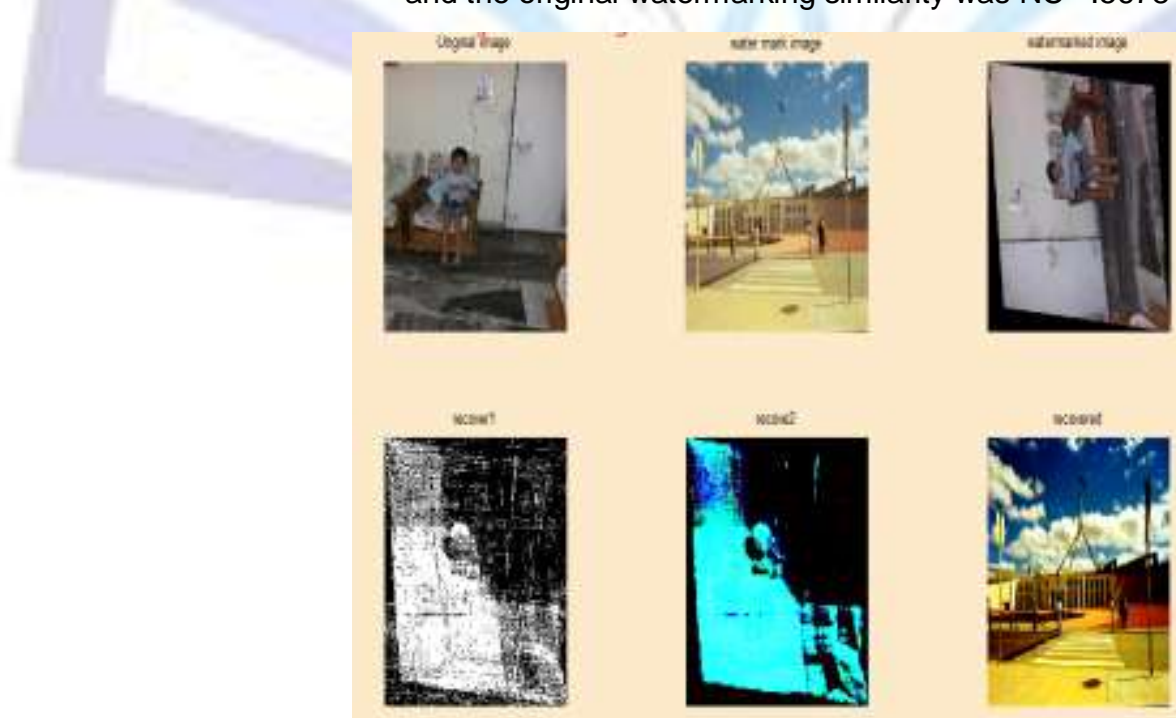

Figure 5 indicated that there were no visual differences between host image and the image embedded Watermark. And the watermarking had good imperceptibility. The peak signal to noise ratio was PSNR $=21.2035$, the extracted watermark and the original watermarking similarity was NC $=0.789$ 


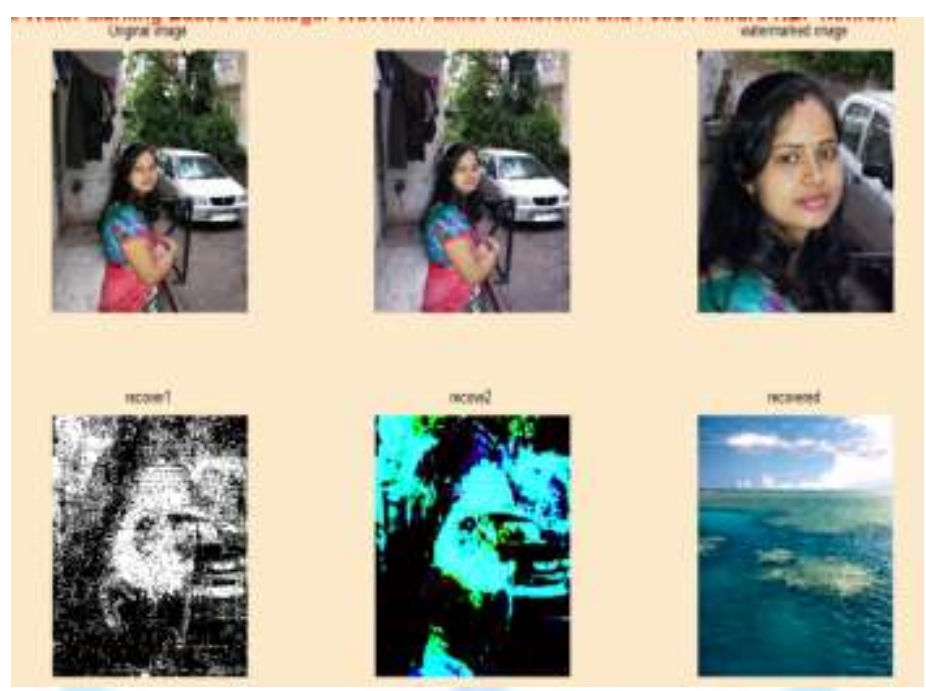

Figure 3 indicated that there were no visual differences between host image and the image embedded Watermark. And the watermarking had good imperceptibility. The peak signal to noise ratio was PSNR $=26.2035$, the extracted watermark and the original watermarking similarity was $\mathrm{NC}=.8765$

Table 1 gives the information about performance of Lena image in watermarking.

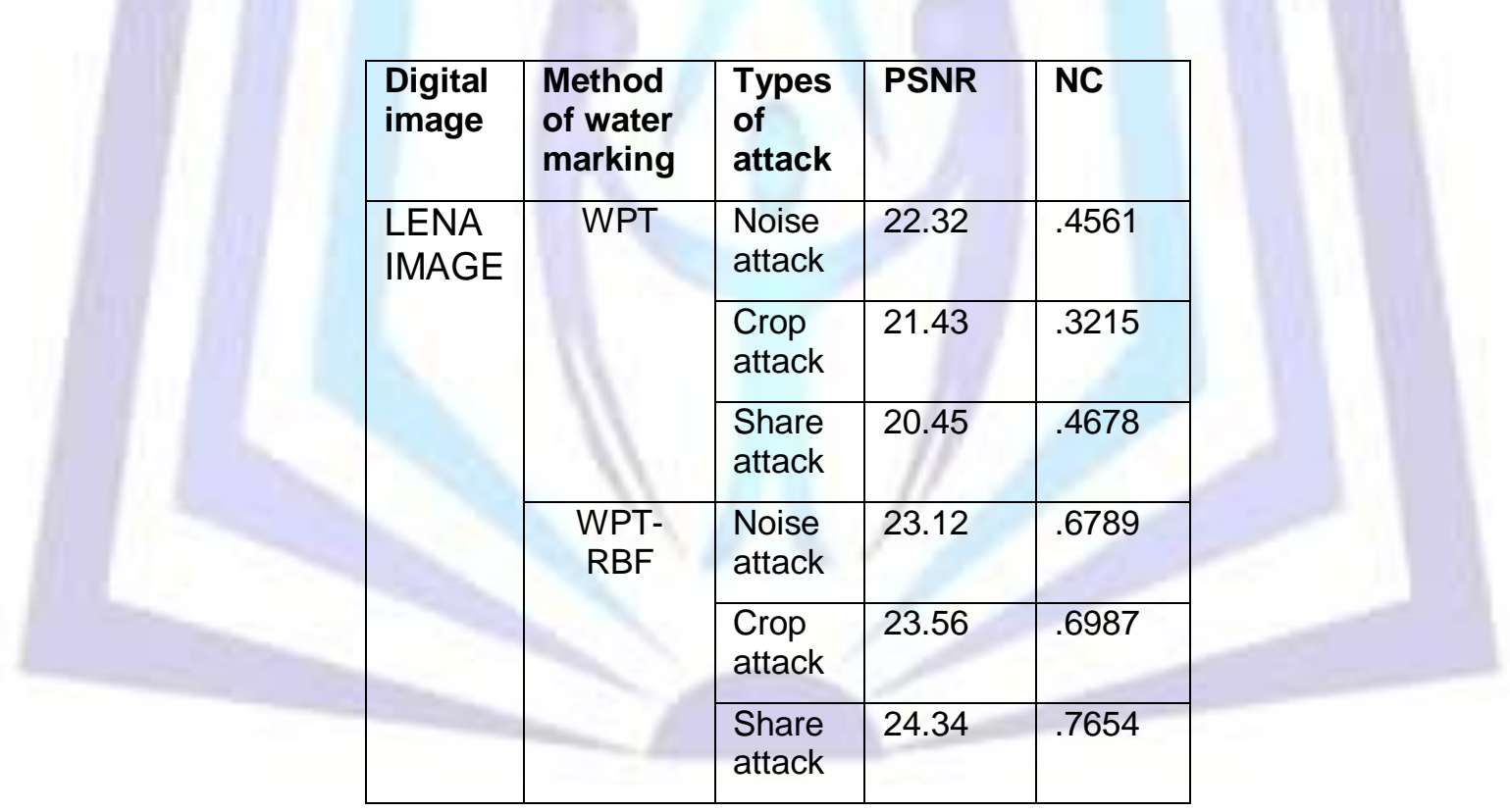


Table 2 gives the information about performance of baby image in watermarking.

\begin{tabular}{|c|c|c|c|c|}
\hline $\begin{array}{l}\text { Digital } \\
\text { image }\end{array}$ & $\begin{array}{l}\text { Method } \\
\text { of water } \\
\text { marking }\end{array}$ & $\begin{array}{l}\text { Types } \\
\text { of } \\
\text { attack }\end{array}$ & PSNR & NC \\
\hline \multirow[t]{6}{*}{$\begin{array}{l}\text { BABY } \\
\text { IMAGE }\end{array}$} & \multirow[t]{3}{*}{ WPT } & $\begin{array}{l}\text { Noise } \\
\text { attack }\end{array}$ & 20.32 & .3561 \\
\hline & & $\begin{array}{l}\text { Crop } \\
\text { attack }\end{array}$ & 21.43 & .3215 \\
\hline & & $\begin{array}{l}\text { Share } \\
\text { attack }\end{array}$ & 22.45 & .5678 \\
\hline & \multirow[t]{3}{*}{$\begin{array}{l}\text { WPT- } \\
\text { RBF }\end{array}$} & $\begin{array}{l}\text { Noise } \\
\text { attack }\end{array}$ & 24.12 & .7789 \\
\hline & & $\begin{array}{l}\text { Crop } \\
\text { attack }\end{array}$ & 22.56 & .6598 \\
\hline & & $\begin{array}{l}\text { Share } \\
\text { attack }\end{array}$ & 23.34 & .4654 \\
\hline
\end{tabular}

Table 3 gives the information about performance of baba image in watermarking

\begin{tabular}{|l|c|l|l|l|}
\hline $\begin{array}{l}\text { Digital } \\
\text { image }\end{array}$ & $\begin{array}{l}\text { Method of } \\
\text { water } \\
\text { marking }\end{array}$ & $\begin{array}{l}\text { Types } \\
\text { of } \\
\text { attack }\end{array}$ & PSNR & NC \\
\hline $\begin{array}{l}\text { BABA } \\
\text { IMAGE }\end{array}$ & WPT & $\begin{array}{l}\text { Noise } \\
\text { attack }\end{array}$ & 23.23 & .6135 \\
\cline { 3 - 5 } & $\begin{array}{l}\text { Crop } \\
\text { attack }\end{array}$ & 22.43 & .5132 \\
\cline { 3 - 5 } & $\begin{array}{l}\text { Share } \\
\text { attack }\end{array}$ & 20.54 & .7568 \\
\cline { 3 - 5 } & WPT-RBF & $\begin{array}{l}\text { Noise } \\
\text { attack }\end{array}$ & 25.21 & .9876 \\
\cline { 3 - 5 } & $\begin{array}{l}\text { Crop } \\
\text { attack }\end{array}$ & 23.65 & .8965 \\
\cline { 3 - 5 } & $\begin{array}{l}\text { Share } \\
\text { attack }\end{array}$ & 24.43 & .7890 \\
\hline
\end{tabular}




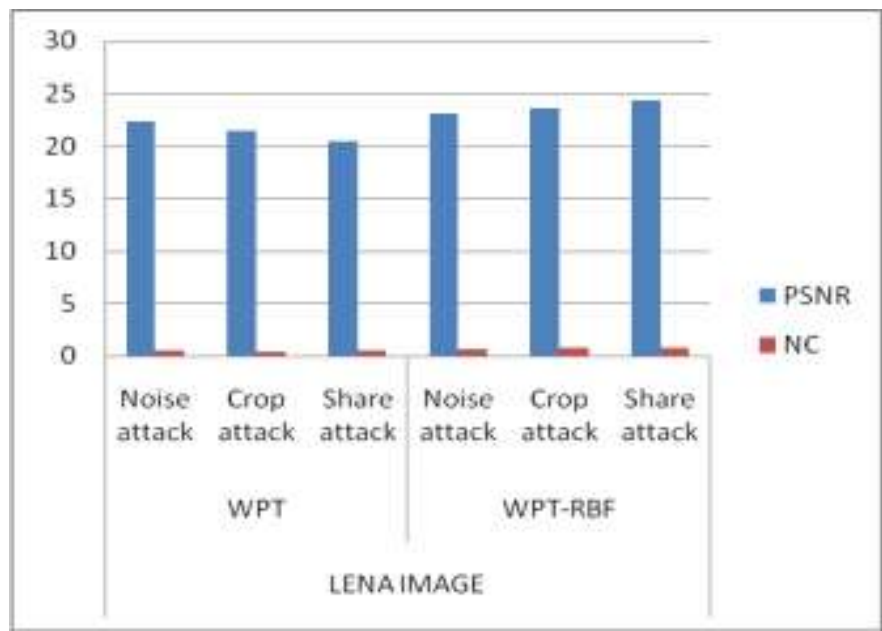

Figure 6 shows that comparative result analysis of Lena image with both method WPT and WPT-RBF in three attack noise attack, crop attack and share attack in terms of PSNR value and NC value

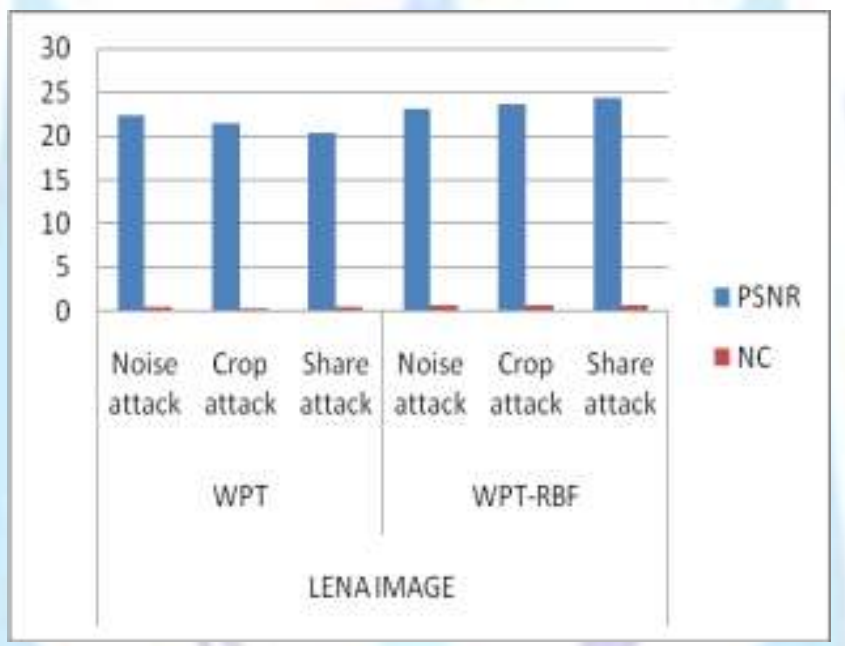

Figure 7 shows that comparative result analysis of Lena image with both method WPT and WPT-RBF in three attack noise attack, crop attack and share attack in terms of PSNR value and NC value

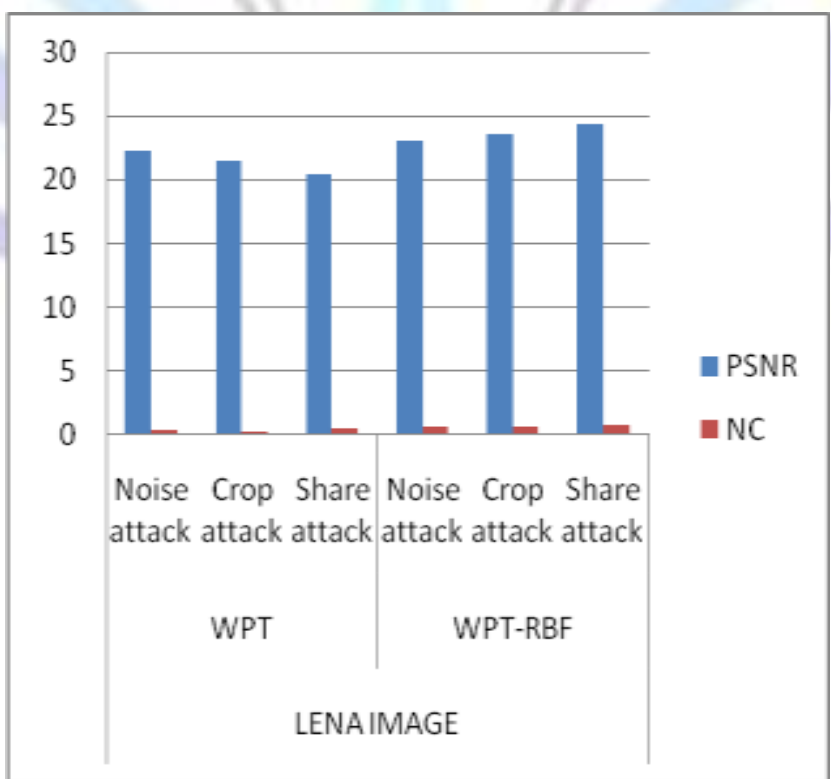

Figure 8 shows that comparative result analysis of Lena image with both method WPT and WPT-RBF in three attack noise attack, crop attack and share attack in terms of PSNR value and NC value 


\section{CONCLUSION AND FUTURE WORK}

In this paper, we proposed the robust digital image watermarking algorithms using WPT transform function using RBF neural network. The novelty of the algorithms is that the watermark is embedded into high and middle frequency components of the host image using Haar wavelet. The advantage of the RBF algorithm is that it provides better NC for median filtering attack compared to WPT technique. That advantage of the proposed technique is that the algorithm is robust to cropping, JPEG compression, salt pepper noise, and rotation attacks compared to WPT technique. The drawback of RBF technique is that the algorithm is vulnerable to median filtering attack. The algorithms can also be applied to video images with some modifications.

\section{REFERENCES:-}

[1] k. K. Wong, c. H. Tse, k. S. Ng, t. H. Lee and I. M. Cheng, "adaptive water marking " in IEEE transaction 1997, PP-8089.

[2] sun jin and yang jing-yu, "quaternion frequency watermarking Algorithm for color images" in IEEE 2010,PP-45-51

[3] qiao baoming, zhang pulin, kang qiao, "a digital watermarking algorithm based on wavelet packet transform and bp Neural network" seventh international conference on computational intelligence and security in 2011,PP 102-106.

[4] mingwei zhao yanzhong dang, " color image copyright protection digital watermarking algorithm based on dwt \& dct " in IEEE transaction 2008 PP 34-40.

[5] ms.kaprebhagyashri and mrs.joshi m.y. " all frequency band dwt-svd robust watermarking technique for color" in IEEE transaction 2011.

[6] haiyan zhao, " algorithm of digital image watermarking technique combined with hvs " in IEEE transaction 2010.

[7] song qiang and zhang hongbin, "color image self-embedding and watermarking based on dwt" in IEEE transaction 2010.

[8] guangmin sun, yao yu, "dwt based watermarking alogritham of color images" in IEEE transaction 2007.

[9] bin zhang, yang xin, xin-xin niu, kai-guo yuan, hui-bai jiang, "a near reversible image watermarking algorithm" in IEEE transaction 2010.

[10] charles way hun fung and walter godoy jr." A novel dwt-svd video watermarking scheme using side view " in IEEE transaction 2011.

[11] yi luo, fei peng, xiaolong li, and bin yang "reversible imagewatermarking based on prediction-error expansion and compensation " in IEEE transaction 2011.

[12] md. Nazmus sakib, syed bahauddin alam ,A b m rafi sazzad, celia shahnaz and shaikh anowarul fattah, "a basic digital watermarking algorithm in discrete cosine transformation domain" in IEEE transaction 2011.

[13] munesh chandra and shikha pandey, "a dwt domain visible watermarking techniques for digital images" international conference on electronics and information engineering in 2010.

[14] rajesh kannan megalingam, mithun muralidharan nair, rahul srikumar, venkat krishnan balasubramanian vineeth sarm venugopala sarma, "performance comparison of novel, robust spatial domain digital image Watermarking with the conventional frequency domain watermarking Techniques" international conference on signal acquisition and processing in 2010.

[15] yu wei, yanling hao and yushen li," a multipurpose digital watermarking algorithm of color image" international conference on mechatronics and automation in 2009.

[16] hang su, chuqing Iv, yanbing ji, yulin wang," a watermarking e-note technique against geometric attacks "2nd international conference on mechanical and electronics engineering in 2010.

[17] qiao baoming, zhang pulin, kang qiao, "a digital watermarking algorithm based on wavelet packet transform and bp neural network" seventh international conference on computational intelligence and security in 2011.

[18] xiao-wei lia, tae-hee namb, seok-ki leea, seok-tae kim, "digital watermarking in transform-domain based on cellular automata transform" in IEEE.

[19] li-jia liu zun-wen he, "an introduction to hyper-complex on meaningful Digital watermarking" in IEEE2010

[20] hang su, chuqing Iv, yanbing ji, yulin wang," a watermarking e-note technique against ks" 2nd international conference on mechanical and electronics engineering in 2010.

[21] kuo-cheng liu," human visual system based watermarking for color images" fifth international conference on information assurance and security in 2009.

[22] munesh chandra", shikha pandel, rama chaudharl," digital watermarking technique for protecting digital images in IEEE trasaction 2010 
[23] stephen mcneill, kerry barton, phil lyver, david pairman," semi-automated penguin counting from digital aerial photographs" in IEEE trasaction 2011.

[24]zheng xiong-bo,zheng xio-wei and sun ming-jian, "a blind digital watermarking alogritham based on wavelet transform" in IEEE trasaction 2011.

[25] mr.manjunatha prasad.r and dr.shivaprakash koliwad, "a robust wavelet-based watermarking scheme for Copyright protection of digital images" second international conference on computing, communication and networking Technologies in 2010.

[26] nirmala palaniswamy, dipesh dugar , dinesh kumar jain and raaja sarabhoje "enhanced blowfish algorithm using bitmap image pixel plotting for security $1 \mathrm{~m}$ provisation" 2nd international conference on education technology and computer in 2010.

[27] D Menghui, Z Jingo "Robust Image Watermarking Algorithm against Geometric Attack Based on BEMD” International Conference on Computer and Communication security IEEE 2009, pp: 36 - 39

[28] T Wenliang “ A Feature -Based Digital Image Watermarking Algorithms Resisting to Geometrical Attack" International Sysposium on Electronic Commerce and Security IEEE 2009, pp: 174 - 178.

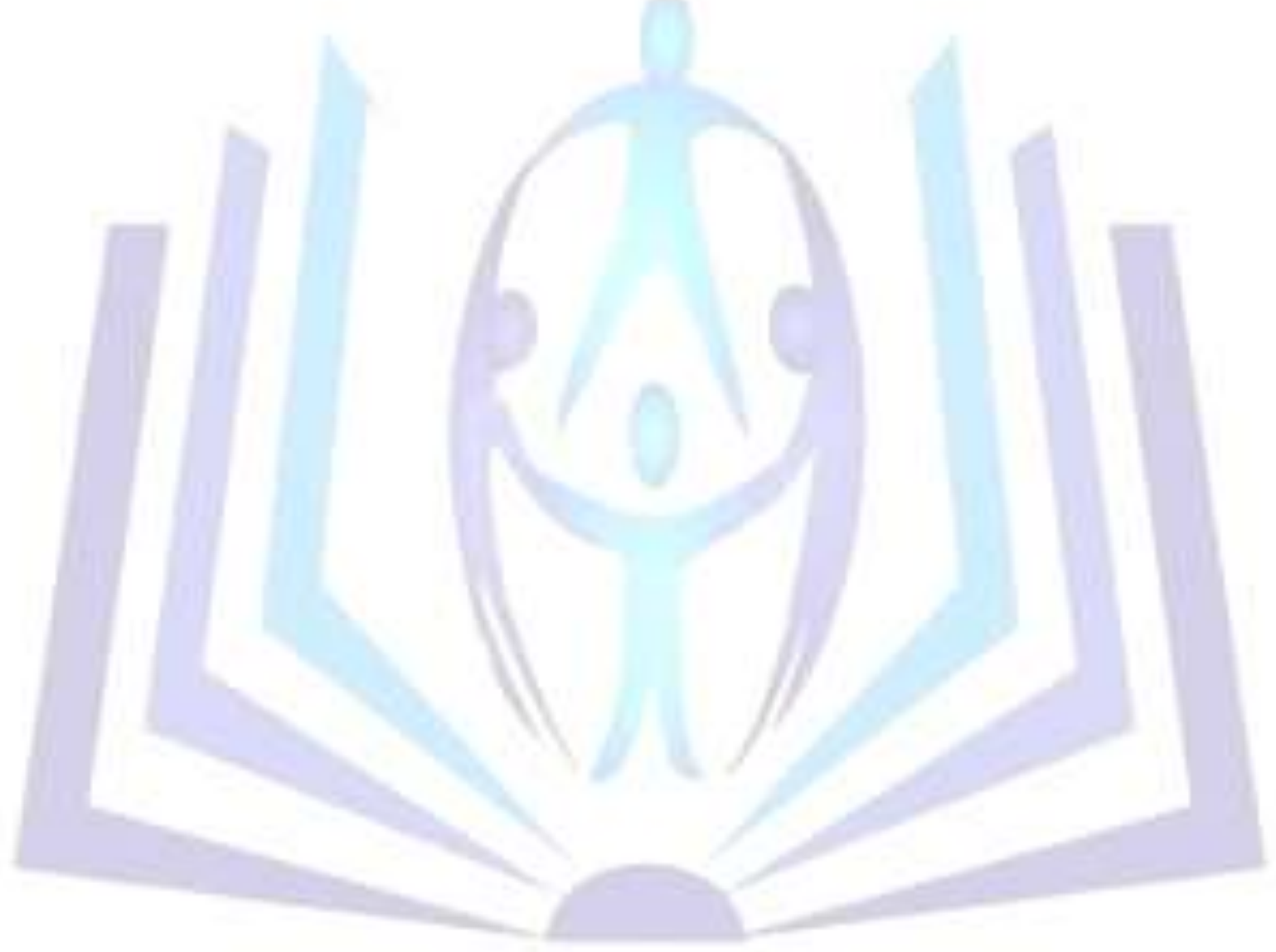

